\title{
JPSE
}

(Journal of Physical Science and Engineering)

\section{The Virtual Lock-in Amplifiers - Accuracy of Measurement Results in Light Transmission Experiments}

\begin{tabular}{l}
\hline Received \\
14 January 2021 \\
Revised \\
13 March 2021 \\
\\
Accepted for Publication \\
01 April 2021 \\
Published \\
29 April 2021
\end{tabular}

L R Prastika ${ }^{1^{*}}$, L Indarti ${ }^{1,2}$, and Hendro ${ }^{3}$

1. SEAMEO Regional Centre for QITEP in Science (SEAQIS), Jl. Diponegoro 12, Bandung, 40115, Indonesia.

2. Center for the Development and Empowerment of Educators and Natural Science Education Personnel (PPPPTK IPA), Jl. Diponegoro 12, Bandung, 40115, Indonesia.

3. Department of Physics, Faculty of Mathematics and Natural Science, Institut Teknologi Bandung, Jl. Ganesha 10, Bandung, 40132, Indonesia.

*E-mail: lintang.r.prastika@ gmail.com

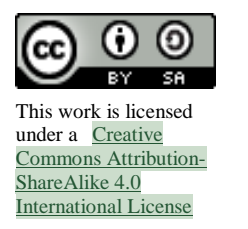

\begin{abstract}
This research focuses on the analysis of the measurement result of the virtual lock-in amplifier (virtual-LIA) in the light transmission experiment as the trial step of developing the virtual-LIA. The virtual-LIA used in this research is designed by using the Vernier sensor DAQ as the data acquisition and the LabVIEW as the programming media. The design of virtual-LIA is based on the mathematical operations of LIA. The type of virtualLIA is a single phase with the capabilities to process the external reference signal. Light transmission experiments are carried out using formazin polymer suspension with turbidity level of $3000 \mathrm{NTU}, 3500 \mathrm{NTU}$, and $4000 \mathrm{NTU}$ as the medium in which light is passed. The accuracy of the measurement results is known by comparing the results of virtual-LIA with real-LIA SR510. The experiments are also carried out in bright and darkroom conditions to determine the ability of virtual-LIA in reducing noise signals. Based on the experiment, the results obtained that the measurement accuracy of the virtual-LIA developed is above $94 \%$ compared to the LIA SR510. Virtual-LIA could measure small signals with and without noise with the average percentage of differences measured between dark and bright conditions is $0.54 \%$.
\end{abstract}

Keywords: Lock in Amplifier, LabVIEW, single phase, external reference, turbid water.

\section{Introduction}

The measurement of the intensity of light sources, such as LEDs using a photodiode, is easy to conduct but quite difficult to obtain an accurate value because we cannot control the number of noise signals. The voltage on the photodiode represents the intensity of LED, but the intensity of the LED is distracted by sunlight and other lights around it so the value measured will not be accurate if we just use the general multimeter.

The way to solve this problem is by using the lock-in amplifier (LIA) instrument. LIA implements a phase-sensitive detection (PSD) method, which can measure very small signals that are covered by noise signals by reducing the signal which does not have the same frequency as the measured signal [1]. Unfortunately, the single-phase LIA instrument is very expensive $(\sim 5000)$ [2]. That condition is raising the researcher's idea to make it into a software-based (virtual) so it can be more economical. Some researchers who have developed the virtual-LIA are Trieu and Duc [1] which makes LIA-based LabVIEW to measure small signals, Stadler et al. [3] that create a virtual lock-in amplifier to measure noise on the resistor, Song et al. [4] uses a virtual-LIA to detect carbon monoxide gas, Ye et al. [5] to make digital LIA LabVIEW-based software to detect power traveling wave signal, Gao et al. [6] who developed the single-channel virtual input dual-phase LIA, Nahar et al. [7] developed the single phase virtual LIA with internal reference based on LabVIEW, and also Madrid and Lopez [8] who developed digital two phase LIA based on LabVIEW.

It is different from the previous studies, the author has developed the simpler type of virtual-LIA, which is the single-phase type with the external reference. This new type of virtual-LIA provides additional alternatives and variations in the development of virtual-LIA. 
This research will focus on the implementation of virtual-LIA that has been developed in light transmission experiments as a trial step for the development of virtual-LIA. The measurement results of the virtual-LIA will be compared with the results of the real-LIA SR510 and oscilloscope to determine the accuracy of virtual-LIA measurements.

\section{Method}

\subsection{Virtual-LIA Design}

The development of this virtual-LIA based on the mathematical operation applied in LIA. LIA is an instrument used to measure small signals covered by noise [9], [10]. LIA processes the periodic signal (such as AC signal) and measures the amplitude and phase of the signal [11]. The working principle of LIA is applying a technique called phase-sensitive detection (PSD) [9], [12]. PSD is a technique to reduce noise by matching the frequency and phase of the noise signal with the reference signal which has been modulated with the input signal. The noise signal which has no equal value of the frequency and phase with the reference signal will be filtered to produce a value of the input signal amplitude. LIA can measure a small periodic signal that is covered by the noise signal amplitude which is much greater than the periodic signal itself [13]-[15].

LIA is composed of the three main parts to processing the periodic signal namely, the reference signal generator, the multiplier, and the low-pass filter (LPF). PSD process occurs in the multiplier and the LPF [12], [15]. Based on the number of PSD, there are two types of LIA namely, single-phase LIA and dual-phase LIA [16]-[18]. In this study, the author has developed a single-phase virtual-LIA using the following basic mathematical operations.

Suppose there is an input signal,

$$
V_{i n}=A \cos \left(\omega_{i n} t+\theta_{i n}\right)
$$

and the reference signal

$$
V_{r e f}=B \cos \left(\omega_{r e f} t+\theta_{r e f}\right)
$$

Both signals are then processed in the PSD through the multiplier

$$
V_{\text {in }} \times V_{\text {ref }}=A \cos \left(\omega_{i n} t+\theta_{i n}\right) \times B \cos \left(\omega_{r e f} t+\theta_{r e f}\right)
$$

Based on the rules of trigonometry, the result of multiplying both signals are as follows

$$
V_{\text {in }} \times V_{\text {ref }}=\frac{1}{2} A B\left[\cos \left\{\left(\omega_{i n}+\omega_{\text {ref }}\right) t+\left(\theta_{\text {in }}+\theta_{\text {ref }}\right)\right\}+\cos \left\{\left(\omega_{i n}-\omega_{r e f}\right) t+\left(\theta_{i n}-\theta_{r e f}\right)\right\}\right]
$$

Frequency of the reference signal should be equal to the frequency of the input signal $\left(\omega_{\text {ref }}=\right.$ $\left.\omega_{\mathrm{in}}\right)$, so that the differences will be equal to zero and generate an equation of PSD signal,

$$
V_{P S D}=\frac{1}{2} A B\left[\cos \left\{2 \omega t+\left(\theta_{\text {in }}+\theta_{\text {ref }}\right)\right\}+\cos \left(\theta_{\text {in }}-\theta_{\text {ref }}\right)\right]
$$

$V_{\mathrm{PSD}}$ then passed to the LPF that will eliminate the trigonometry component that have $2 \omega t$ value and generates a DC signal as the output of LIA, with the equation

$$
V_{P S D}=\frac{1}{2} A B \cos \left(\theta_{i n}-\theta_{r e f}\right)
$$

Equation (6) showed that there are components of the phase difference between the reference and the input. The single-phase LIA needs to be done to adjust the reference phase and resulting $V_{\mathrm{PSD}}$ maximum value. The maximum value of $V$ obtained when the reference phase is equal to the input phase $\left(\theta_{\text {ref }}=\right.$ $\theta_{\mathrm{in})}$.

$$
V_{P S D}=\frac{1}{2} A B
$$




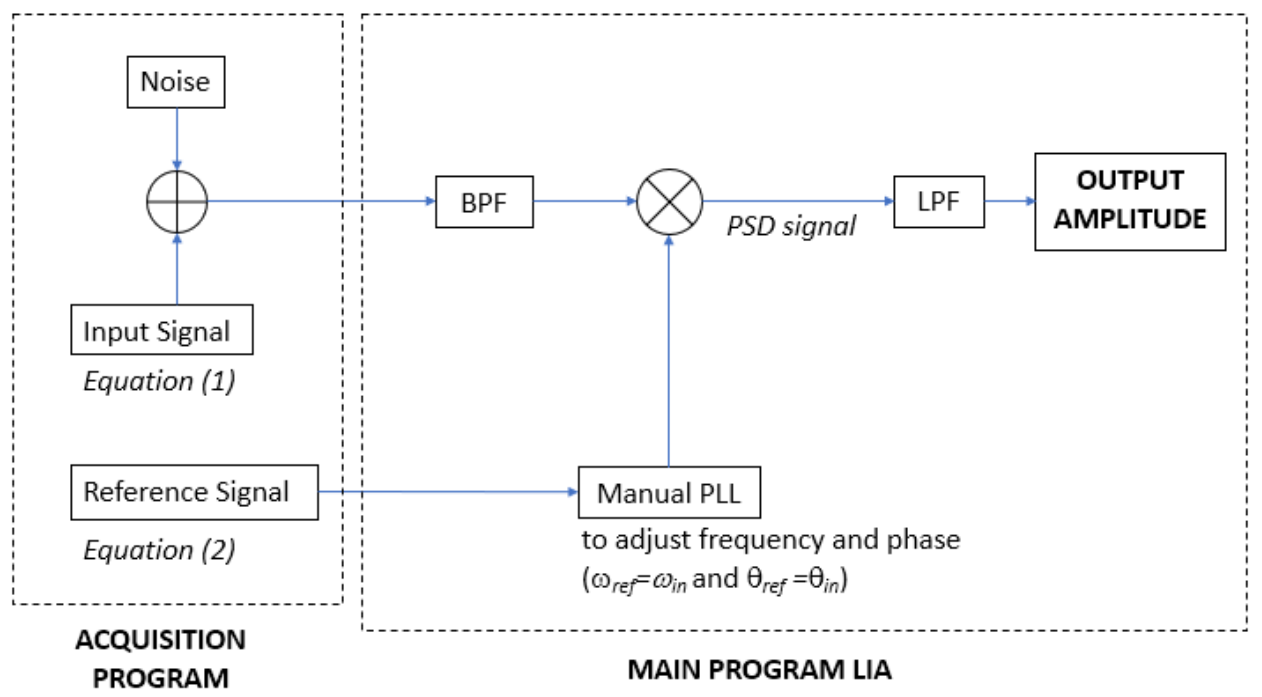

Figure 1. Block diagram of virtual-LIA.

The output is the value of the input signal amplitude $A$, which can be calculated using equation (8) below.

$$
A=\frac{2}{B} V_{P S D}
$$

All the mathematical operations above, then programmed in LabVIEW block diagram uses the LabVIEW function palettes.

Figure 1 shows the general design of the developed virtual-LIA's block. There were two main parts of the virtual-LIA that have been developed, 1) the data acquisition program and 2) the main program. The input signal is covered by noise and reference signal read by data acquisition tools. It transforms the analogue signal from photodiode into digital signal then, using the data acquisition program, forwards it to the LIA main program to be processed.

In the main program, aside from writing all the LIA's mathematical operation in the block diagram, the author uses the manual phase-locked loop (PLL) function to adjust the frequency and phase of a reference signal virtually from LabVIEW and the band-pass filter (BPF) component to reduce noise that covers the input signal. The signal from BPF and manual PLL then passes the PSD process (multiplier and LPF) to result the output signal measured.

\subsection{Experiment}

To find out the accuracy of the measurement results, the virtual-LIA that has been developed then it is tested to the light transmission experiment in turbid water. The transmission value calculated uses the following equation (9).

$$
T=\frac{I_{i}}{I_{0}}
$$

Where $T$ is the light transmission value of light, $I_{0}$ is the intensity of the incident light, and $I_{\mathrm{i}}$ is the light intensity after passing through the medium [19]. In this study, the value of light intensity will be represented by voltage of light detector measured using virtual-LIA. Furthemore, the equation (9) will be,

$$
T=\frac{V_{i}}{V_{0}}
$$

where $V_{0}$ is the detector voltage before the light passes through the sample and $V_{\mathrm{i}}$ is the detector voltage after the light has passed through the sample [20], [21]. 
In this experiment, virtual-LIA is used to measure the voltage of the SP-8 ML photodiode sensor as a light detector. This sensor could capture light which has wavelength between $450 \mathrm{~nm}$ to $1050 \mathrm{~nm}$. This wide range of capturing makes the sensor more sensitive to capture a variety of light besides of the main light source. This capability is needed in testing the virtual-LIA to see its ability to filter noise and produce accurate output.

The SP-8 ML photodiode sensor reads the signal from a green laser as a light source. It has a wavelength of about $532 \mathrm{~nm}$. This laser is used because it is a coherent light source.

The turbid water used in this experiment is the formazine polymer suspension with turbidity levels of 3000 NTU, 3500 NTU, and 4000 NTU. The purpose of using the water sample with high turbidity is to produce a small signal from a light source. Variation of the turbidity value is applied to see the consistency of virtual-LIA in measuring small signals. Furthermore, this small signal is read by the photodiode sensor. This condition is needed to see the accuracy of the virtual-LIA in measuring small signals that are covered by noise. The circuit scheme of the light transmission experiment in turbid water is shown in Figure 2a.

Just like the actual LIA device, virtual-LIA will only process periodic signals. Therefore, the laser beam which is a DC signal needs to be manipulated into a periodic signal. The process of manipulating DC signals into periodic signals is called chopping (signal labelling).

Signal labelling in this experiment do not use the mechanical labelling, but electronic labelling. The electronic labelling process is done by adding the $\mathrm{BC} 108$ transistor component to the circuit. The emitter pin is connected to the ground, the collector pin is connected to the laser, and the base pin is connected to the signal generator as the periodic signal generator so that in low frequencies the laser light will blinked. The periodic signal from this signal generator is also used as the LIA reference signal.

Moreover, the data collection process is conducted. The turbid water sample is poured onto the cuvette then placed parallel between the laser and the detector (Figure 2b). The data collected in this experiment is the value of the photodiode voltage as a representation of the light intensity received by the photodiode. The voltage when the cuvette is empty (not filled with turbid water) is recorded as $V_{0}$ and the voltage after the cuvette is filled with turbid water is recorded as $V_{\mathrm{i}}$. The values of $V_{\mathrm{i}}$ and $V_{0}$ were measured in two conditions, dark and bright, to see the virtual-LIA's ability to reduce noise around the system. In dark conditions, the system is covered with a black cloth so that there will be no other light can be detected except the laser beam. In bright conditions, the system is not covered with black cloth that the photodiode will receive other light around the laser beam. This voltage value is measured by the virtual-LIA as well as LIA SR510 and Gw Instek GDS-1102A oscilloscope as a comparison. The accuracy of the virtual-LIA measurement results is calculated using a percentage compared to the LIA SR510 measurement results.

To ensure that the input signal is covered with the noise, it is necessary to observe the graphic form displayed by the virtual-LIA. The advantage of virtual-LIA is that it can display direct graphic form, while real LIA cannot. The graphical display of the input signal from virtual-LIA is compared with oscilloscope to determine the quality and suitability of virtual-LIA in displaying the graph.

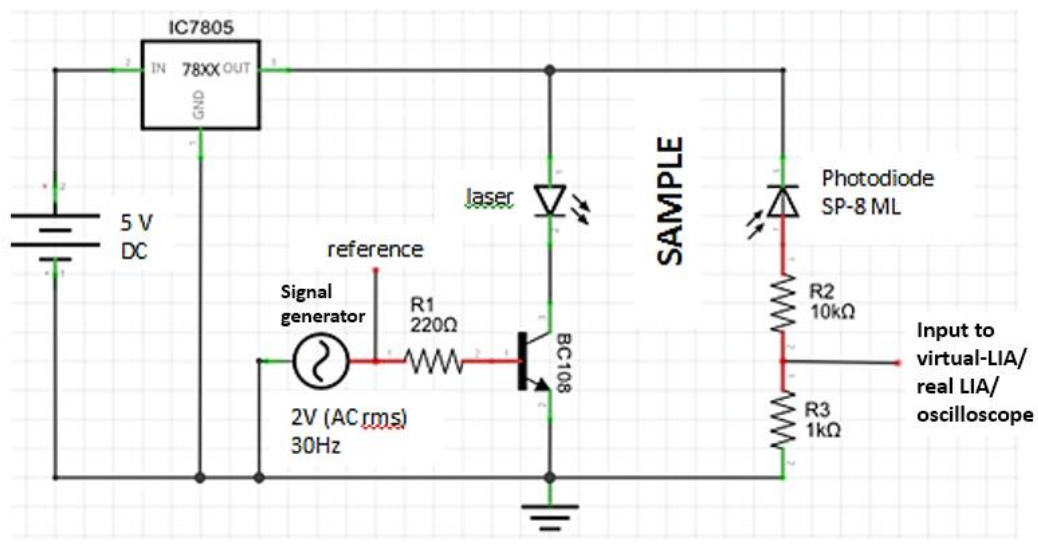

(a)

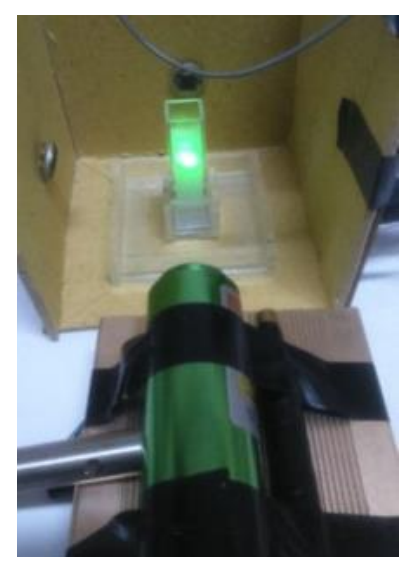

(b)

Figure 2. (a) The circuit scheme of the light transmission experiment in turbid water, (b) The system of laser, cuvette, and photodiode. 


\section{Result and Discussion}

\subsection{Graphic Form}

The graph of the input signal after passing through the dark and bright turbid water is observed to see the signal when it is covered by noise. Graph form of virtual-LIA and oscilloscope readings for each turbidity level is shown in Tables $1-4$.

Table 1 shows the graphical form of the input signal when passing through an empty cuvette. The amplitude value is about 0.2 volts. In bright conditions the signal is covered by noise, it can be seen from the virtual-LIA graph that the position of the trough changes from zero (in dark conditions) increases to 0.05 . The graphic shape on the oscilloscope shows the same condition, there is a change towards the positive $y$-axis in the position of the trough. The weakness of this oscilloscope is that it cannot show the scale value on the $x$-axis and $y$-axis clearly.

Table 2 shows the signal amplitude decreases when it passes through turbid water by 3000 NTU to around 0.03 volts. Noise disturbed conditions can be seen from the graph in bright conditions, that the trough position change from -0.01 to 0.06 .

Based on the graph in Table 3 it is known that the input signal has the same amplitude value, which is around 0.03 volts after passing through turbid water with a turbidity level of 3500 NTU. It can also be seen that the input signal is disturbed by noise in bright conditions.

Similarly, in Table 4, based on the graph that is displayed by the virtual-LIA and the oscilloscope input signal amplitude value after going through the murky waters of the NTU 4000 was about 0.03 and the signal covered by noise in bright conditions.

This condition in Tables 1-4 shows that the input signal is covered by the noise and the virtualLIA can function properly in displaying the signal graph. In addition, by comparing the graphs in Tables $1-4$, it can also be seen that the turbidity of water can make the input signal smaller. It can be seen from the amplitude of the signal in Tables 2-4 which is smaller than the amplitude of the signal in Table 1. Both conditions are needed to test the virtual-LIA's ability to filter out noise in small input signals.

Table 1. Graphical display of the signal from the SP-8 ML detector for empty cuvette in dark and bright conditions.

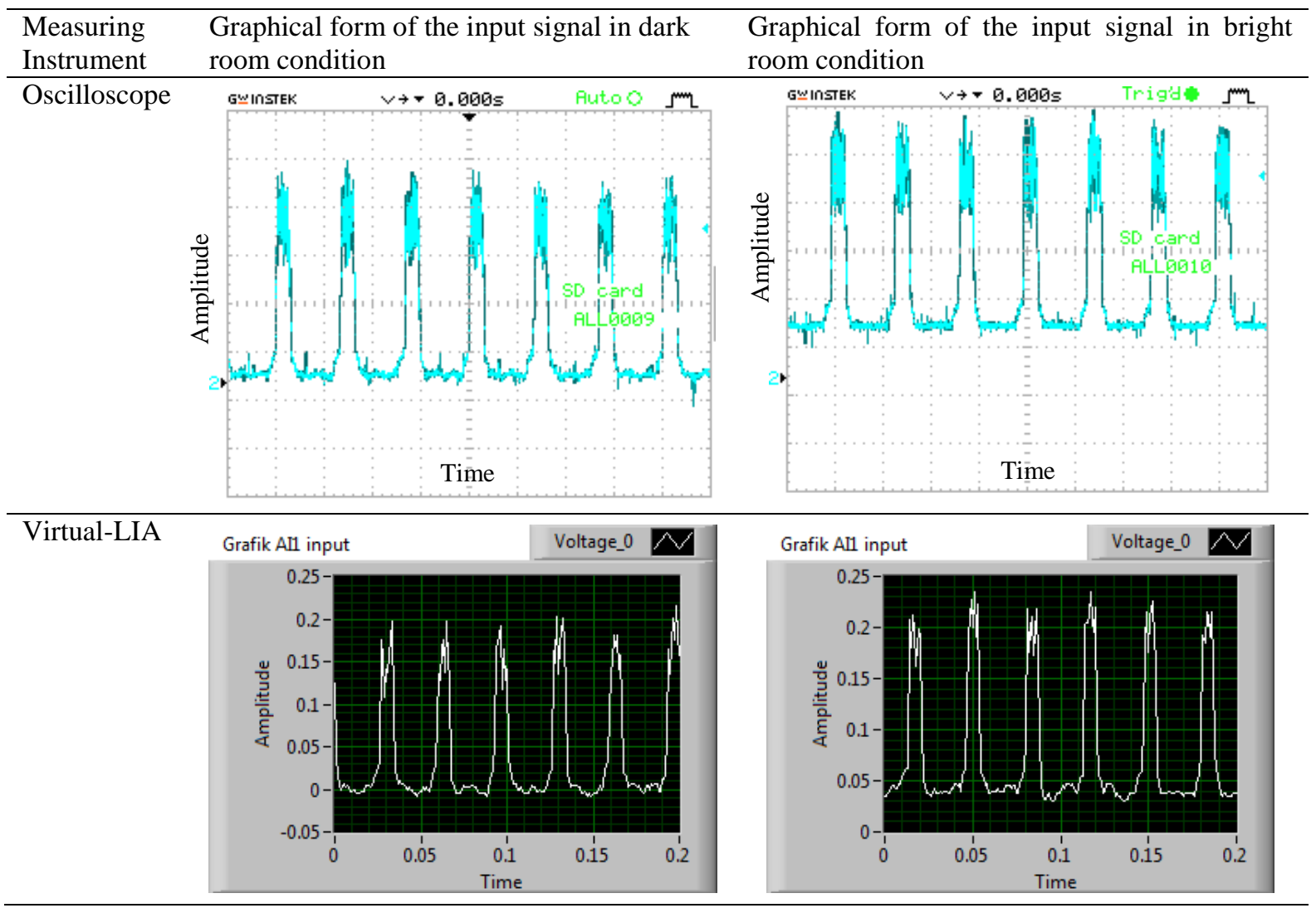


Table 2. Graphical display of the signal from the SP-8 ML detector for 3000 NTU turbidity level in dark and bright conditions.

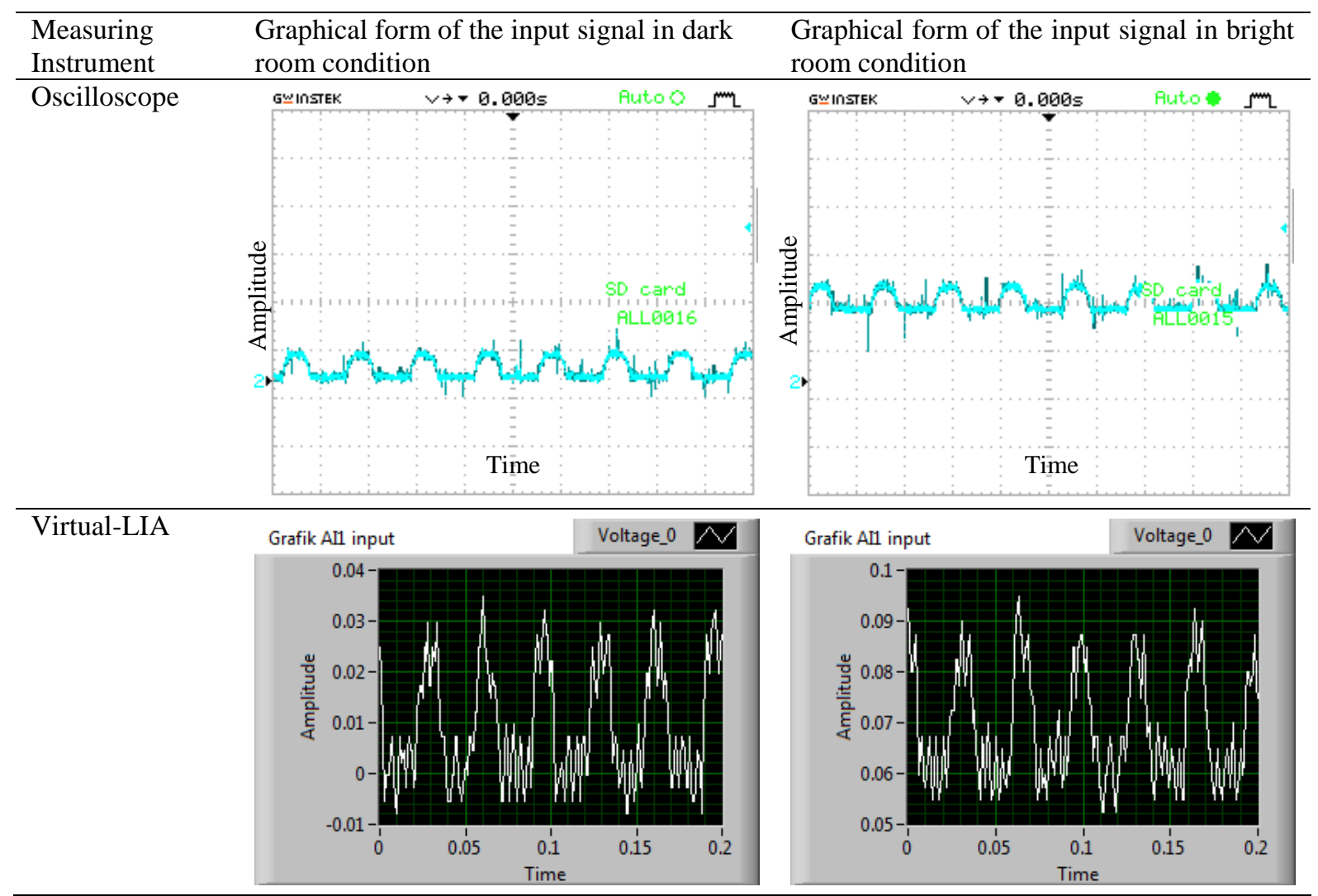

Table 3. Graphical display of the signal from the SP-8 ML detector for 3500 NTU turbidity level in dark and bright conditions.

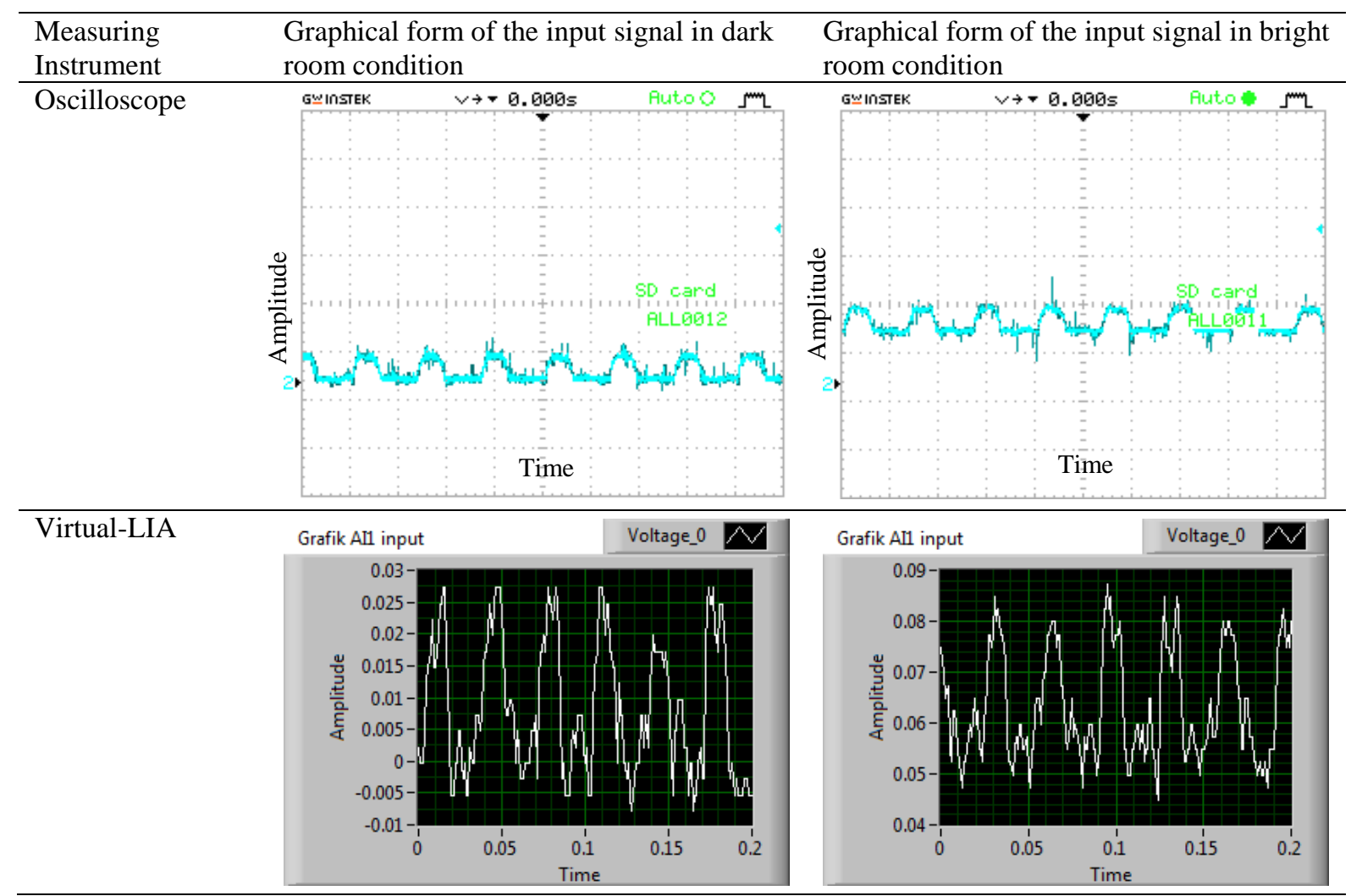


Table 4. Graphical display of the signal from the SP-8 ML detector for 4000 NTU turbidity level in dark and bright conditions.

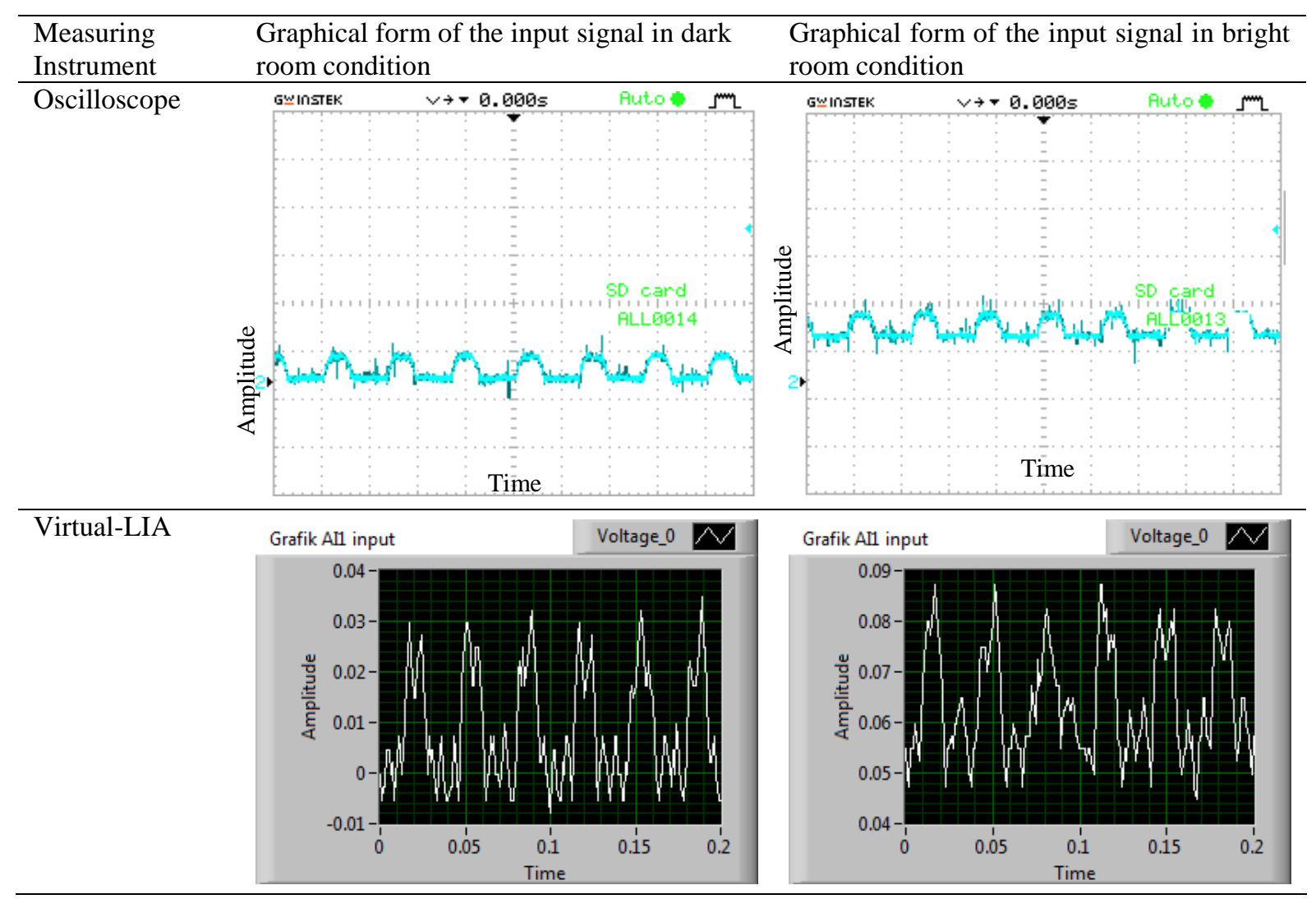

\subsection{Values of $V_{0}$ and $V_{i}$}

Values of $V_{0}$ and $V_{\mathrm{i}}$ are measured by using the virtual-LIA, LIA SR510, and an oscilloscope in dark and bright room conditions. Before comparing the turbid water transmission values measured by the virtualLIA and LIA SR510, we may see the first a comparison of the voltage measurement results in dark and bright conditions for each measuring instrument. This aims to determine the ability of the measuring instrument to reduce noise covered the input signal. The measurement results of the oscilloscope, virtual-LIA, and LIA SR510 are shown in Tables 5-7.

Table 5 shows the difference between the photodiode voltage measurement by the oscilloscope in light and dark conditions reaches an average value of $307 \%$. This indicate that the input signal is covered by much greater value of noise and the oscilloscope cannot filter the noise.

Table 5. Voltage measurement of photodiode SP-8 ML by oscilloscope.

\begin{tabular}{|c|c|c|c|c|c|c|c|}
\hline \multirow{2}{*}{ Sample } & \multicolumn{2}{|c|}{$V_{0}$ RMS (volt) } & \multirow{2}{*}{$\begin{array}{c}\text { Differences } \\
(\%)\end{array}$} & \multirow{2}{*}{ Sample } & \multicolumn{2}{|c|}{$V_{i}$ RMS (volt) } & \multirow{2}{*}{$\begin{array}{c}\text { Differences } \\
(\%)\end{array}$} \\
\hline & Dark & Bright & & & Dark & Bright & \\
\hline \multirow{3}{*}{$\begin{array}{l}\text { Empty } \\
\text { cuvette }\end{array}$} & 0.043 & 0.096 & 123 & $3000 \mathrm{NTU}$ & 0.013 & 0.086 & 561 \\
\hline & 0.043 & 0.077 & 79 & $3500 \mathrm{NTU}$ & 0.012 & 0.070 & 483 \\
\hline & 0.044 & 0.096 & 118 & $4000 \mathrm{NTU}$ & 0.012 & 0.069 & 475 \\
\hline & \multicolumn{2}{|c|}{ Average } & \multicolumn{5}{|c|}{$307 \%$} \\
\hline
\end{tabular}

Table 6. Voltage measurement of photodiode SP-8 ML by virtual-LIA.

\begin{tabular}{|c|c|c|c|c|c|c|c|}
\hline \multirow{2}{*}{ Sample } & \multicolumn{2}{|c|}{$V_{0}$ RMS (volt) } & \multirow{2}{*}{$\begin{array}{c}\text { Differences } \\
(\%)\end{array}$} & \multirow{2}{*}{ Sample } & \multicolumn{2}{|c|}{$V_{i}$ RMS (volt) } & \multirow{2}{*}{$\begin{array}{c}\text { Differences } \\
(\%)\end{array}$} \\
\hline & Dark & Bright & & & Dark & Bright & \\
\hline \multirow{3}{*}{$\begin{array}{l}\text { Empty } \\
\text { cuvette }\end{array}$} & 0.048 & 0.048 & 0.00 & $3000 \mathrm{NTU}$ & 0.0092 & 0.0092 & 0.00 \\
\hline & 0.048 & 0.047 & 2.08 & $3500 \mathrm{NTU}$ & 0.0087 & 0.0088 & 1.15 \\
\hline & 0.048 & 0.048 & 0.00 & $4000 \mathrm{NTU}$ & 0.0087 & 0.0087 & 0.00 \\
\hline & \multicolumn{2}{|c|}{ Average } & \multicolumn{5}{|c|}{$0.54 \%$} \\
\hline
\end{tabular}


Table 7. Voltage Measurement of photodiode SP-8 ML by LIA SR510.

\begin{tabular}{|c|c|c|c|c|c|c|c|}
\hline \multirow{2}{*}{ Sample } & \multicolumn{2}{|c|}{$V_{0}$ RMS (volt) } & \multirow{2}{*}{$\begin{array}{c}\text { Differences } \\
(\%)\end{array}$} & \multirow{2}{*}{ Sample } & \multicolumn{2}{|c|}{$V_{i}$ RMS (volt) } & \multirow{2}{*}{$\begin{array}{c}\text { Differences } \\
(\%)\end{array}$} \\
\hline & Dark & Bright & & & Dark & Bright & \\
\hline \multirow{3}{*}{$\begin{array}{l}\text { Empty } \\
\text { cuvette }\end{array}$} & 0.048 & 0.048 & 0.00 & 3000 NTU & 0.0088 & 0.0088 & 0.00 \\
\hline & 0.049 & 0.048 & 2.04 & $3500 \mathrm{NTU}$ & 0.0088 & 0.0088 & 0.00 \\
\hline & 0.049 & 0.049 & 0.00 & $4000 \mathrm{NTU}$ & 0.0088 & 0.0088 & 0.00 \\
\hline & \multicolumn{2}{|c|}{ Average } & \multicolumn{5}{|c|}{$0.34 \%$} \\
\hline
\end{tabular}

Based on Table 6, the measurement results of the photodiode by virtual-LIA are relatively stable, both in dark and light conditions, with the average value of differences is $0.54 \%$. This value differs. It is only $0.20 \%$ compared to the LIA SR510 measurement results in Table 7 . These data indicate that the $V_{0}$ and $V_{\mathrm{i}}$ measured by virtual-LIA are very close to the LIA SR150 measurement results. These results prove that the developed virtual-LIA can filter noise well so that it can produce accurate data. This condition is in accordance with the main function of the actual LIA instrument.

\subsection{Light Transmission Value}

Furthermore, to reassure the accuracy of the virtual-LIA's measurements, $V_{0}$ and $V_{\mathrm{i}}$ are processed to determine the transmission value of each turbid water sample. The transmission value is calculated using equation (10). The value of turbid water transmission is shown in Table 8.

From the turbid water transmission value in Table 8 , it is known that each water turbidity level has almost the same transmission value. This shows that water with turbidity levels of 3000 NTU, 3500 NTU, and 4000 NTU has the same density and can also be included to a substance with high density because the transmission value was relatively small. The high-density value indicates that the distance between particles of the substance is so close. If the high-density substance exposed by the light, most of the light rays will be absorbed by the particles that make up the substance and a few other are transmitted through the substance so that the transmission value of these substances will be small.

The voltage value read by the virtual-LIA in both light and dark conditions is relatively the same. This again shows that the developed virtual-LIA has functioned well in filtering noise. The final step is to calculate the accuracy of virtual-LIA compared to the LIA SR510. The percentage of virtual-LIA measurement accuracy is shown in Table 9. From Table 9 the developed virtual-LIA already has very good accuracy with a value of above $94 \%$ compared to the measurement results of the LIA SR510.

\section{Conclusion}

From the experiment and analysis of the measurement results, it is known that the virtual-LIA developed by the author has functioned well such as the real LIA instrument. The virtual-LIA can filter out noise in the input signal with an average percentage difference between measurements in light and dark conditions of $0.54 \%$. Virtual-LIA can also measure the value of turbid water transmission with a high accuracy of above $94 \%$. Virtual-LIA also has advantages over the LIA SR510 instrument, which has a function like an oscilloscope that can display signal graphs.

Table 8. Values of light transmission by turbid water.

\begin{tabular}{lcccccc}
\hline \multirow{2}{*}{ Sample } & \multicolumn{6}{c}{ Transmission Value $\left(T=V_{i} / V_{0}\right)$} \\
\cline { 2 - 7 } & Dark & Light & Average & Dark & Light & Average \\
\cline { 2 - 7 } & 0.19 & 0.19 & 0.190 & 0.18 & 0.18 & 0.180 \\
\hline 3000 NTU & 0.18 & 0.19 & 0.185 & 0.18 & 0.17 & 0.175 \\
\hline 3500 NTU & 0.18 & 0.18 & 0.180 & 0.18 & 0.18 & 0.180 \\
\hline 4000 NTU & 0.18 &
\end{tabular}

Table 9. The percentage accuracy of the measurement of turbid water transmission compared by virtual-LIA to LIA SR510.

\begin{tabular}{lccc}
\hline Sample & $V_{i} / V_{0}$ Virtual-LIA & $V_{i} / V_{0}$ LIA SR510 & Accuracy to the LIA SR510 \\
\hline 3000 NTU & 0.190 & 0.180 & $94.7 \%$ \\
\hline 3500 NTU & 0.185 & 0.175 & $94.6 \%$ \\
\hline $4000 \mathrm{NTU}$ & 0.180 & 0.180 & $100 \%$ \\
\hline
\end{tabular}




\section{References}

[1] P. Q. Trieu and N. A. Duc, "Implementation of the digital phase-sensitive system for low signal measurement," VNU J. Sci., Math.-Phys., vol. 24, pp. 239-244, 2008.

[2] L. Guillemot et al., "Watt-level mid-infrared continuous-wave Tm: YAG laser operating on the 3H4 $\rightarrow$ 3H5 transition," Optical Mat., vol. 101, p. 109745, 2020.

[3] A. W. Stadler, A. Kolek, Z. Zawiślak, and A. Dziedzic, "Noise measurements of resistors with the use of dual-phase virtual lock-in technique," Metrology and Measurement System, vol. 22, no. 4, pp. 503-512, 2015.

[4] N. Song et al., "A mid-infrared carbon oxide detection system based on virtual lock-in amplifier technology," J. Optoelectronics Laser, vol. 25, pp. 2343-2349, 2014.

[5] B. Ye, F. Chen, and M. Li, "The digital lock-in amplifier for detecting the power traveling wave signal," Int. J. Signal Process., Image Process. Pattern Recognition, vol. 8, no. 4, pp. 361-374, 2015.

[6] Z. Gao, H. Zheng, L. Li, F. Chen, and F. Guo, "A single channel input virtual dual-phase lock-in amplifier," in The Int. Society for Optical Eng. Proc. SPIE, 2011, p. 81971F.

[7] W. S. Nahar, R. Umbarawati, Hendro, "Simulasi virtual lock-in amplifier fase tunggal dengan rujukan internal berbasis LabVIEW," in Pros. Sem. Kontribusi Fis., 2017.

[8] G. M. Madrid and J. M. A. Lopez, "Lock-in amplifier based on virtual instrumentation," Treball de Fi de Grau, Facultat de Física, Universitat de Barcelona, 2016.

[9] Z. Gao, H. Zheng, L. Li, F. Chen, and F. Guo, "A single channel input virtual dual-phase lock-in amplifier," in 2011 Int. Conf. Optical Instruments and Technol.: Optical Systems and Modern Optoelectronic Instruments, vol. 8197, 2011, p. 81971F.

[10] I. J. Bhagyajyothi, P. Bhaskar, C. S. Parvathi, "Design and development of advanced lock-in amplifier and its application," Sensors \& Transducers, vol. 153, no. 6, pp. 22-28, 2013.

[11] J. J. Vandenbussche, P. Lee, and J. Peuteman, "On the accuracy of digital phase sensitive detectors implemented in FPGA technology," IEEE Transactions on Instrumentation and Measurement, vol. 63, no. 8, pp. 1926-1936, 2014.

[12] J. J. Vandenbussche, J. Peuteman, and P. Lee, "Development of a low-cost accurate phase measurement system," in 2014 Inf. Comm. Technol. Innov. Appl. (ICTIA), 2014, pp. 1-7.

[13] K. Achtenberg, J. Mikołajczyk, and Z. Bielecki, "FET input voltage amplifier for low frequency noise measurements," Metrology and Measurement Systems, vol. 27, no. 3, pp. 531-540, 2020.

[14] K. Zhang et al., "Application of digital quadrature lock-in amplifier in TDLAS humidity detection," in AOPC 2017: Optical Spectroscopy and Imaging, vol. 10461, 2017, p. 1046109.

[15] W. Weiming, M. Shengyuan, and L. Xiangdong, "Detection of weak modulation signal by digital phase-locked amplifier," J. Phys.: Conf. Ser., vol. 1345, no. 4, p. 042096, 2019.

[16] Q. Wang, H. Zheng, and M. Jiang, "Implementation of digital lock-in amplifier based on system generator," in 2016 IEEE Int. Conf. Signal and Image Process. (ICSIP), 2016, pp. 636-640.

[17] J. Sinlapanuntakul, P. Kijamnajsuk, C. Jetjamnong, and S. Chotikaprakhan, "Digital lock-in amplifier based on soundcard interface for physics laboratory," J. Phys.: Conf. Ser., vol. 901, no. 1, p. 012065, 2017.

[18] L. E. Bengtsson, "A microcontroller-based lock-in amplifier for sub-milliohm resistance measurements," Rev. Sci. Instruments, vol. 83, no. 7, p. 075103, 2012.

[19] W. Wahyuni, N. Novita, Fajriani, and Hendro, "Rancang bangun alat ukur transmisi dan absorpsi cahaya berbasis arduino dan LabVIEW," in Pros. Simposium Nas. Inov. Pembelajar. Sains 2015 (SNIPS 2015), 2015, pp. 105-108.

[20] G. L. Jiang, H. Yang, R. Li, and P. Kong, "A new algorithm for a high-modulation frequency and high-speed digital lock-in amplifier," Measurement Sci. Technol., vol. 27, no. 1, p. 015701, 2015.

[21] J. Wang, Z. Wang, X. Ji, J. Liu, and G. Liu, "A simplified digital lock-in amplifier for the scanning grating spectrometer," Rev. Sci. Instruments, vol. 88, no. 2, p. 023101, 2017. 